\title{
Los modos de contar en los programas magacines de la radio argentina: descripción y análisis de los géneros radiofónicos
}

\section{Storytelling approaches in magazine radio programs in Argentina: description and analysis of radio genres}

\author{
Martínez-Costa Pérez, M. P. y Müller, M. J. ${ }^{1}$ \\ Recibido: 17-04-2018 - Aceptado: 14-06-2018 \\ DOI: https://doi.org/10.26441/RC17.2-2018-A7
}

\begin{abstract}
RESUMEN: El presente artículo estudia los modos de contar de los programas magacines de máxima audiencia de la radio argentina. Se analizan los contenidos de los cuatro programas más escuchados y de fuerte competencia entre ellos al inicio de la presente década. Argentina tiene un mercado radiofónico consolidado, con una penetración del $70 \%$ y cuyo pico de audiencia o prime time se localiza entre las 10.00 y las 12.00 horas. Mediante el análisis de contenidos de 160 horas de programación de radio se persigue estudiar el uso de los géneros en los programas líderes del prime time de la radio argentina, según sean de información y opinión, ficción y entretenimiento, o participación de la audiencia. A partir de la frecuencia de aparición y la duración, se mostrará la importancia asignada en la OM/AM y la FM a cada uno de los géneros radiofónicos, así como el grado de integración en el discurso de continuidad que caracteriza a los magacines.
\end{abstract}

Palabras clave: narrativa radiofónica; géneros radiofónicos; radio en Argentina; programas magacines.

\begin{abstract}
The study focuses on storytelling techniques applied in prime-time radio magazines in Argentina. The article analyzes the four leading audience programs at the beginning of this decade. Argentina has a concentrated market, radio penetration reaches $70 \%$ and prime time is between 10:00 and 12:00 AM. By means of content analysis of 160 hours of radio programming, use of different radio genres is analyzed in a wide range of prime time programs from news and opinion to fiction and entertainment, including audience participation. The comparative study of frequency and length of each genre, in different programs and different broadcast bands, shows the relative importance of each and the level of use along the continuity which define magazine as a radio format.
\end{abstract}

Key words: radio narrative; radio genres; radio in Argentina; radio programs.

1 María del Pilar Martínez-Costa es Doctora en Comunicación Pública, Profesora Titular del Departamento de Proyectos Periodísticos de la Facultad de Comunicación de la Universidad de Navarra, en donde imparte las asignaturas Información Radiofónica y Narrativa Radiofónica. marcosta@unav.es, https://orcid.org/00000002-6564-0207

María José Müller es Doctora en Comunicación y Profesora a cargo de Lenguaje Sonoro y Taller de Producción Sonora en la Licenciatura en Comunicación en la Universidad Austral (Argentina). Es profesora en la materia Calidad en la Comunicación en la Maestría en Gestión de Contenidos de la Escuela de Posgrados. mmuller@ austral.edu.ar, https://orcid.org/0000-0003-2870-3261 


\section{Introducción}

El programa magacín es la estrella de la programación en la radio generalista de los últimos 30 años, tanto en el mercado de la radio española como latinoamericana (López Vigil, 1997; Cuní, 1999; Martí, 2000; Rodero 2011). A lo largo de estas décadas este tipo de programa se ha erigido en la columna vertebral de la oferta radiofónica, alcanzando las mayores cuotas de audiencia y convirtiéndose en un programa maratón de larga duración en el esquema de franjas horarias de producción.

El magacín nace como un tipo de "programa-tractor" de la radio generalista para la captura de grandes audiencias y se transforma rápidamente en el motor de su oferta de contenidos:

El magacín es un programa de larga duración, emplazado en las franjas horarias importantes para la captación masiva de audiencia, dirigido por un/a conductor/a "estrella", con contenidos de actualidad, dotado de importantes equipos profesionales para la redacción, locución y producción, contando con una amplia presencia de colaboradores y expertos que enriquecen los contenidos del programa (MartínezCosta y Díez Unzueta, 2005, 186).

De esta forma, la identidad de la emisora queda reflejada en los contenidos y el ritmo de presentación en la antena del magacín (Flemming, 2010, 64). Su estructura combina información, opinión, entretenimiento y espectáculo, con un cambio de ritmo constante para adaptarse a los tiempos y ritmos de escucha de la audiencia. Un conductor con fuerte personalidad, y gran presencia delan- te del micrófono, facilita la identificación y fidelidad de la audiencia, que junto a los colaboradores y secciones fijas ayudan a construir la unidad del programa en el relato cambiante de la actualidad del día. Todas las funciones son importantes, desde el operador técnico al colaborador, pasando por el productor y el presentador, ya que se trata de un trabajo coral. Conformar un buen equipo con diferentes funciones y opiniones genera "el equilibrio y la variedad" que necesita un programa para funcionar (Portugal y Yudchak, 2008, 35).

Aunque estas características esenciales se mantienen, esta fórmula de éxito se ha adaptado a las nuevas formas y necesidades de la escucha. Más ligado a la información de actualidad en un principio, el entretenimiento ha ganado terreno en los magacines de la radio. Los tratamientos narrativos de la información del día, producidos a partir del ritmo de la actualidad, se mezclan ahora con el relato en continuidad y con una producción más creativa y cuidada, con el fin de construir espacios capaces de "generar nuevos valores” (Haye, 1995). El conductor sigue siendo el pilar sobre el que se construye el programa, pero ganan terreno las voces de los expertos y, con ellos, los géneros de autor y de opinión. Ha sido el programa estrella de la radio generalista y ahora empieza a formar parte de los tramos de mañana de la radio especializada temática, que busca entretener y ganar audiencia (MartínezCosta y Müller, 2015; Moreno, Amoedo y Martínez-Costa, 2017). Más aún, en un momento de expansión y consolidación de la radio online (Martínez-Costa, 
Moreno y Amoedo, 2018), el magacín sigue siendo el programa que ayuda a construir la marca de la emisora.

Estos cambios percibidos en la ideación y puesta en antena del magacín radiofónico hacen interesante el estudio de sus modos de contar, lo que en ámbito anglosajón se denomina storytelling, que consiste en el arte de contar historias que empaticen con la audiencia, generando confianza y fidelizando la escucha. En concreto, aquí se analizará el tipo de géneros radiofónicos o estructuras narrativas utilizadas en cada una de las horas de los programas magacín de las 4 emisoras más escuchadas de la radio argentina, un mercado radiofónico importante, con una penetración del $90 \%$ de la población, y cuyo pico de audiencia se localiza entre las 10.00 y las 12.00 horas.

\section{Objeto y método}

Mediante la descripción y análisis de contenido de los géneros radiofónicos ${ }^{2}$ utilizados, este artículo se propone estudiar su uso en los programas líderes del prime time de la radio argentina y su integración en el discurso de continuidad que caracteriza a los programas magacines. Los programas analizados son los que, según las mediciones de audiencia realizadas por IBOPE Argentina, acumulaban la mayor audiencia en octubre de 2010, cuando se realizó la captura de datos de la muestra, a saber: El Oro y el Moro del grupo Radio 10 y Hola Chiche de Radio Mitre, que son representativos de la OM/AM; y ¿Cuál Es? y Terapia Despareja que pertenecen a la FM y se emiten por Rock \& Pop y Pop, respectivamente ${ }^{3}$.

Este estudio realizado en perspectiva pretende identificar los elementos comunes que caracterizan la forma de contar de los magacines, que luego se han mantenido con variaciones narrativas menores en las siguientes temporadas radiofónicas, aun cuando los programas hayan cambiado de denominación y presentadores.

Algunas de las preguntas que se plantean responder son las siguientes: ¿Hay diferencias en las formas de contar de los programas del prime time de la OM/AM y la FM? ¿Es posible identificar el uso de géneros radiofónicos en estos programas que aparentemente fluyen con la naturalidad de la conversación no planificada? ¿Qué géneros informativos, de entretenimiento y de participación predominan en cada programa y en cada banda de emisión? ¿Se pueden identificar estrategias narrativas comunes en los magacines de máxima audiencia?

En el momento de realizar el estudio, los cuatro programas eran magacines

2 Se define como géneros radiofónicos "los modelos de representación de la realidad que otorgan estructura y orden a los contenidos de la radio para conseguir la creación de sentido por parte del emisor y la interpretación de sus mensajes por parte del receptor" (Martínez-Costa y Díez Unzueta, 2005, 97). En este sentido, los géneros radiofónicos subraya Haye, son el "molde de una estrategia" comunicativa de la radio $(2003,93)$. Véase también Martínez-Costa y Herrera Damas (2004 y 2005).

3 Según los datos de la empresa IBOPE Media Argentina, la cuota de audiencia de los programas estudiados en 2010 era: El Oro y el Moro, Radio 10, del grupo Indalo (en 2010 del grupo Infobae), 1,91; Hola Chiche, Radio Mitre, del Grupo Clarín, 1,33; Terapia Despareja, Pop Radio, también hoy del Grupo Indalo, 1,54; Cuál Es?, Rock \& Pop, hoy del grupo Moneta (en 2010 de CIE), 1,60. 
liderados por un conductor protagónico, conocido también por su participación en otros medios, sobre todo la televisión. Contaban con un amplio equipo de colaboradores especializados, que amenizaban la mañana de la radio con intervenciones frecuentes. Se definían, y aún se definen, como programas de entretenimiento y actualidad, con variedad de contenidos y voces, y que ocupaban lo que se denomina la "segunda mañana" de la radio, que transcurre de las 9.00 a las 13.00 horas. Este fue el tramo horario seleccionado para el análisis.

Se estudiaron 10 ediciones de cada programa, es decir 40 horas de cada programa y 160 horas de programa- ción en total. Se identificaron los géneros utilizados y se cronometraron los tiempos de duración de cada uno de ellos, de modo de identificar con más precisión las fronteras entre unos y otros, así como señalar la importancia de cada género a partir de la frecuencia de aparición y la duración asignada.

Para desarrollar el análisis se elaboró una matriz en la que se clasificó a los géneros en 3 categorías, según sean de información y opinión, ficción y entretenimiento, y participación de la audiencia. Los géneros que finalmente se analizaron fueron los que se utilizan con mayor frecuencia, quedando descartados otros de uso esporádico.

Tabla 1. La matriz para el estudio de los géneros

\begin{tabular}{|c|c|c|c|c|c|}
\hline \multirow{9}{*}{ Géneros } & \multirow{9}{*}{ Tipos } & \multirow{4}{*}{ Información y Opinión } & Crónica & Frecuencia & Tiempo \\
\hline & & & Entrevista & & \\
\hline & & & Comentario & & \\
\hline & & & Tertulia & & \\
\hline & & \multirow{3}{*}{ Ficción y Entretenimiento } & Acting & & \\
\hline & & & Imitación & & \\
\hline & & & Cuento de humor & & \\
\hline & & \multirow{2}{*}{ Participación de la audiencia } & Concurso & & \\
\hline & & & Mensaje & & \\
\hline
\end{tabular}

Fuente: Elaboración propia.

A través de este análisis de contenido se persigue conocer qué tipo de géneros predominan, qué frecuencia de uso se constata en cada programa por hora y en total, y qué duración tienen en aquellos casos en se puedan medir. El objetivo es identificar la variedad y el uso que se hace de los principales géneros radiofónicos en el prime time. Entre los de información y opinión, se inclu- yeron en la matriz de análisis la crónica, el comentario, la entrevista y la tertulia. Aunque la noticia es un género informativo relevante, se decidió descartarla porque solo aparece en "estado puro" en los servicios informativos de las emisoras, que no son estudiados en este trabajo. Dentro de los géneros de ficción y entretenimiento se estudiaron el acting, la imitación y el cuento de 
humor. Finalmente, los géneros de participación de la audiencia analizados fueron los concursos y mensajes, las dos formas más comunes de interacción con la audiencia en los programas del prime time. Tanto en este caso como en los géneros de ficción y entretenimiento se analizó sólo la frecuencia de aparición en cada programa, debido a las dificultades que suponía determinar su duración exacta, dado que muchas veces se presentan de modo no planificado, intermitente, y en medio de otros contenidos y géneros.

El análisis sobre el uso de los géneros reveló en qué medida predomina la información, la opinión o el entretenimiento en cada programa, y a su vez, permitió identificar algunas diferencias entre las franjas horarias, los programas y los tipos de emisoras según sean de OM/AM o FM.

\section{Características generales del merca- do de la radio en la Argentina}

La radio continúa siendo un medio de fuerte penetración en la Argentina, incluso a partir de los cambios tecnológicos y de la escucha a través de diferentes soportes y plataformas. Según la Encuesta Nacional de Consumos Culturales 2017, producida por el SInCA, el $70 \%$ de la población escucha radio y el $62 \%$ usa el aparato receptor tradicional. Los argentinos escuchan, en promedio, entre 3 y 4 horas de radio por día.

El mercado de la radio en la Argentina está volcado fundamentalmente a la actividad privada y se financia sobre todo a través de la publicidad. Además, cuenta con el Servicio Oficial de Radiodifusión (SOR), conformado por un conjunto de emisoras públicas, sostenidas por el Estado. A esta estructura de radios comerciales y públicas, se suman las radios comunitarias, entre las que hay religiosas, rurales, escolares, universitarias.

En cuanto a la programación, la radio generalista siempre se destacó en el mercado argentino: contenidos variados, un conductor "estrella", diversidad de voces que acompañan (locutores, columnistas, cronistas, etc.), un modelo de bloques y un servicio informativo fuerte. La radio especializada, a su vez, también tiene una fuerte presencia, aunque no hay muchas emisoras FM de formato musical cerrado ni radios temáticas. Es decir, el modelo de radio especializada que se impone en la Argentina combina un estilo musical definido con programas de contenidos variados que proponen un modelo especializado híbrido alternando siempre música y palabra.

\subsection{Las emisoras y los programas ana- lizados}

Las dos emisoras de AM estudiadas en este trabajo, Radio Mitre y Radio 10, se alternan en el liderazgo de audiencia desde hace dos décadas. Mitre pertenece al Grupo Clarín y cumplió 90 años en 2015 , lo que la convierte en una de las radios de mayor trayectoria, ligada a los comienzos de la radiodifusión en la Argentina. Radio 10 nació en 1998, de la mano del periodista y empresario Daniel Hadad, y un año después ya se había posicionado primera.

En cuanto a las FM analizadas, Rock \& Pop es una emisora emblemática de la 
historia de la FM argentina. Destacó desde el principio por una identidad definida, asociada al rock, de rica producción artística, que fue gestando un oyente fiel y comprometido. Está viviendo momentos de grandes cambios a partir de la marcha de Mario Pergolini en 2012, conductor insignia desde su nacimiento. Por su parte, Pop Radio nació en 2004 y se posicionó muy rápido en los primeros puestos del ranking. Es una FM más re- conocida por sus programas y conductores, que por su diseño musical.

En la Tabla 2 puede observarse el $\mathrm{ra}$ ting promedio de lunes a domingo de 6.00 a 24.00 (2010), donde se evidencia que las radios analizadas ocupan los primeros lugares entre la audiencia. Como se adelantó, en este trabajo se seleccionaron los 4 programas que lideraban el rating en el horario del prime time, ubicado a las 10.00 horas.

Tabla 2. Rating promedio de 6.00 a 24.00 (2010)

\begin{tabular}{|r|l|r|}
\hline \multicolumn{3}{|c|}{ Rating promedio 2010- 6:00 a 24:00 } \\
\hline 1 & Radio 10 - AM 710 & 1,91 \\
\hline 2 & LA 100 - FM 99.9 & 1,80 \\
\hline 3 & Pop Radio - FM 101.5 & 1,60 \\
\hline 4 & Rock \& Pop - FM 95.9 & 1,54 \\
\hline 5 & Metro - FM 95.1 & 1,35 \\
\hline 6 & Mitre - AM 790 & 1,33 \\
\hline 7 & Los 40 Principales - FM 105.5 & 1,25 \\
\hline 8 & Vale - FM 97.5 & 1,08 \\
\hline 9 & Mega - FM 98.3 & 1,08 \\
\hline 10 & Radio Disney - FM 94.3 & 1,05 \\
\hline 11 & Aspen - FM 102.3 & 0,97 \\
\hline 12 & Continental - AM 590 & 0,91 \\
\hline 13 & TKM Radio -FM 103.7 & 0,78 \\
\hline 14 & Radio La Red - AM 910 & 0,76 \\
\hline 15 & Radio Nacional - AM 870 & 0,39 \\
\hline
\end{tabular}

A continuación se detallan esquemáticamente las características formales de los programas analizados.

Nombre del programa: Hola Chiche

Días de emisión: lunes a viernes

Horario: 9.00 a 13.00

Emisora: Radio Mitre AM 790

\begin{tabular}{|r|l|r|}
\hline \multicolumn{3}{|c|}{ Rating promedio 2010- 6:00 a 24:00 } \\
\hline 16 & Radio Uno - 103.1 & 0,31 \\
\hline 17 & Del Plata - AM 1030 & 0,29 \\
\hline 18 & Blue - FM 100.7 & 0,23 \\
\hline 19 & La 2X4 - FM 92.7 & 0,17 \\
\hline 20 & Milenium - FM 106.7 & 0,15 \\
\hline 21 & Nacional Folklórica - FM 98.7 & 0,15 \\
\hline 22 & ESPN - FM 107.9 & 0,13 \\
\hline 23 & El Mundo - AM 1070 & 0,08 \\
\hline 24 & Nacional Clásica - FM 96.7 & 0,08 \\
\hline 25 & Imagina - FM 104.3 & 0,06 \\
\hline 26 & Radio Belgrano - AM 950 & 0,04 \\
\hline 27 & Radio de la Ciudad - AM 1110 & 0,02 \\
\hline Encendido AM+FM & 22,78 \\
\hline Otras FM & 2,08 \\
\hline Otras AM & 0,66 \\
\hline
\end{tabular}

Conductor: Samuel "Chiche" Gelblung Colaboradores: Cristina Wargon, Marcela Giorgi (locutora), Horacio Pagani (deporte), Silvia Naishtat (economía), Mauro Szeta (policiales), Rodrigo Lucich (espectáculos), Ariel Tarico (humor). 
Hola Chiche era un magacín generalista que integraba variedad y cantidad de contenidos en un todo liderado por un conductor de fuerte personalidad y estilo: Chiche Gelblung. El relato se construía alrededor de Gelblung, que hilaba los contenidos con un gran sentido de la improvisación, combinada con una producción periodística fuerte y un equipo de colaboradores que cubría todos los temas de interés. Hola Chiche era un programa en continuidad, solo interrumpido cada media hora por el servicio informativo del Mitre Informa Primero y por las tandas publicitarias.

Nombre del programa: El Oro y el Moro

Días de emisión: lunes a viernes

Horario: 9.00 a 12.00

Emisora: Radio 10 AM 710

Conductor: Oscar "El Negro" Gonzalez Oro

Colaboradores: Valeria Mirabella (locutora), Chiche Almozny (deporte), Eduardo Feinman (política), Claudio Zin (Salud), Laura Ubfal (espectáculos), Alexis Garbarz (tecnología).

El Oro y el Moro combinaba heterogeneidad de contenidos y voces en un producto con una identidad bien definida por la impronta de su conductor y por el sonido característico de Radio 10. Se abordaban todas las temáticas haciendo eje en la actualidad pero mucha veces dejando lo urgente para el servicio informativo de la radio. González Oro le imprimía un ritmo acelerado al programa, con entradas breves pero intermitentes de los columnistas, entrevistas, móviles y mensajes de oyentes. Como buen conductor "bastonero", se concentraba en distribuir el juego y recuperar permanentemente el entretenimiento y el humor, muy característico de él.

Nombre del programa: Cuál Es?

Días de emisión: lunes a viernes

Horario: 9.00 a 13.00

Emisora: Rock and Pop FM 95.9

Conductor: Mario Pergolini

Co-conductores: Eduardo De la

Puente, Marcelo Gantman

(deportes)

Colaboradores: Gustavo Olmedo (música), Diego Miller (personajes-humor), Santiago Bluggerman (guionista), Maximiliano Peñalver (videojuegos) Guillermo Hernández (cine) Leo Regueira (automovilismo), Jorge Halperín (curiosidades), Mariano Sena (cronista).

Cuál Es? era un magacín especializado en un formato musical que contenía diferentes temáticas y voces con eje en un tipo de contenido: la música rock. Fue el programa principal de Rock \& Pop durante casi 25 años, con un conductor referente que arrastraba grandes audiencias. Se construía sobre la base del diálogo permanente, de estilo improvisado, entre los conductores, colaboradores e invitados. Fue un programa con abundante pre-producción, centrado en la música, el humor y una estética cuidada.

Nombre del programa: Terapia

Despareja

Días de emisión: lunes a viernes

Horario: 9.00 a 13.00

Emisora: Pop 103.5

Conductores: Santiago del Moro, Analía Franchín 
Colaboradores: Rolo Villar (humorista), Diego Brancatelli (deporte), Sara Whertein (curiosidades).

Terapia Despareja era un magacín de entretenimiento con variedad de contenidos, voces principales y secundarias y numerosas secciones. Trabajaba mucho sobre noticias atemporales, curiosidades y temas ajenos a la agenda periodística del día. Proponía una mesa de diálogo permanente entre los conductores y los colaboradores. El humor ocupaba un lugar central en Terapia Despareja, igual que la participación de la audiencia, apelando constantemente a los oyentes a través de mensajes de voz, correos electrónicos y concursos. Su conductor principal, Santiago del Moro, es un referente de la televisión, que aportaba al programa su estilo cálido e informal.

\section{El uso de los géneros de informa- ción y opinión para contar la actuali- dad del día}

Si observamos el modo en el que los programas líderes empleaban los géneros, lo primero que se evidencia es que los 4 programas desarrollan una propuesta diferenciada en relación a su uso, como lo muestra el Gráfico 1.

Gráfico 1. Uso de los géneros de información y opinión por programa

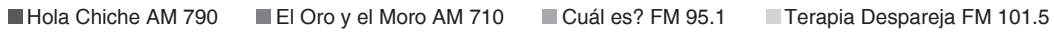

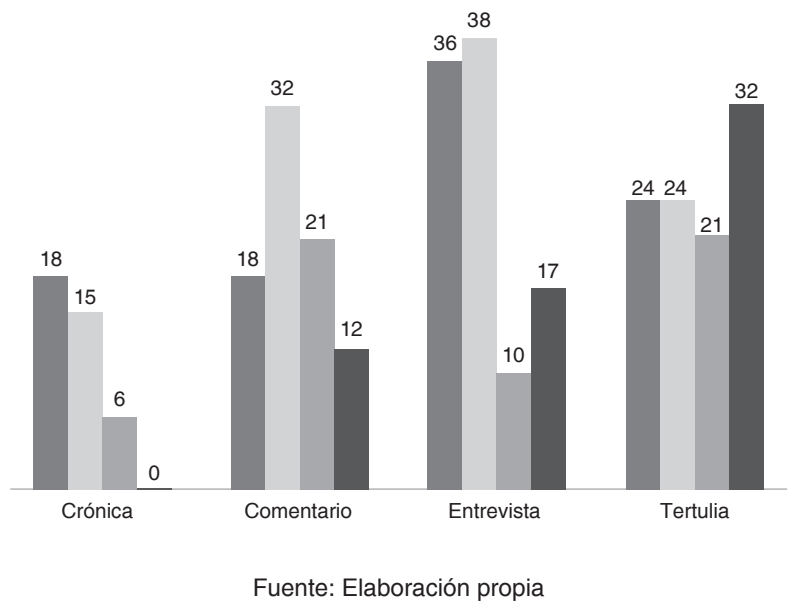

Para Hola Chiche y El Oro y el Moro, los programas representativos de la OM/ AM, el género más empleado es la entrevista, con 36 y 38 entrevistas sobre el total de la muestra. La tertulia es igual de importante para ambos, con 24 cada uno. Hay mayor diferencia en el uso que hacen del comentario, que El Oro y 
el Moro emplea de forma más frecuente que Hola Chiche. La crónica es un género claramente más propio de la $\mathrm{OM} /$ AM, si bien resulta ser el género menos utilizado por los 4 programas del prime time estudiados. En cuanto al uso de géneros en la FM, el género predominante es la tertulia aunque, para ¿Cuál Es?, también lo es el comentario. Terapia Despareja es el programa que más uso hace de la tertulia y el que menos emplea la crónica y el comentario. Se puede concluir entonces que cada programa tiene su propio criterio en el uso de los géneros de información y opinión, aunque la OM/AM y la FM muestran algunas similitudes entre sí. La crónica y la entrevista son más características de la OM/AM y la tertulia es el género que usan regularmente los cuatro programas, mientras que el comentario es el género que muestra un uso más dispar.

Si se compara el uso de los géneros por programa, se observa que para Hola Chiche la entrevista es el género más utilizado, como lo muestra el Gráfico 2.

Gráfico 2. Uso de los géneros de información y opinión por hora de programa en Hola Chiche

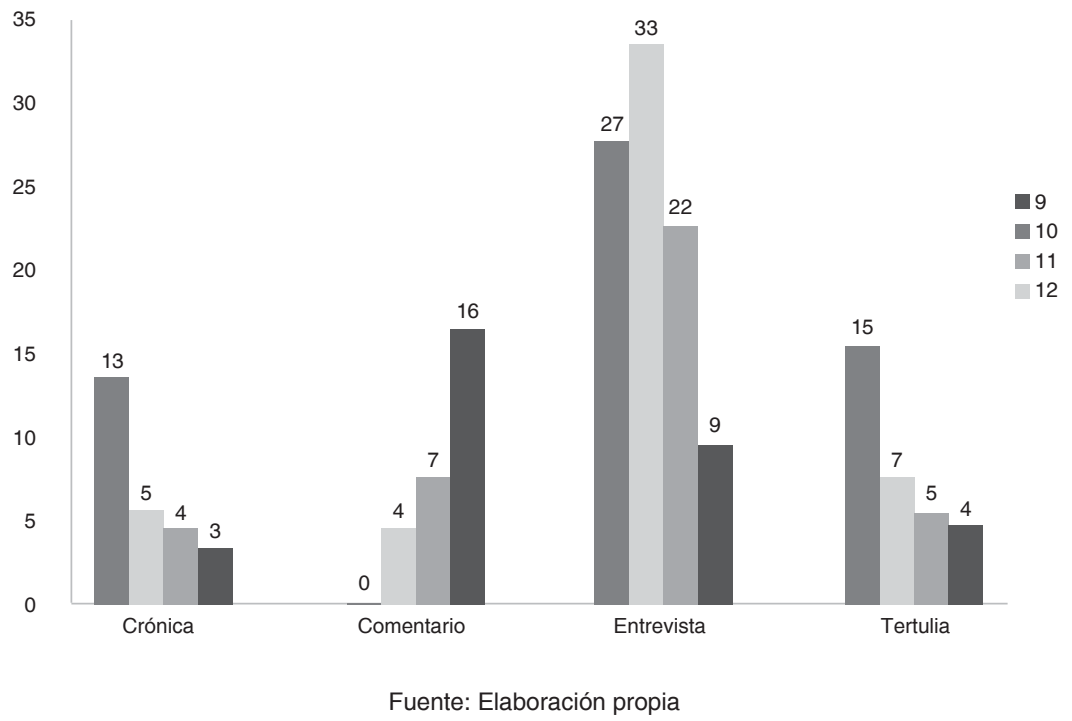

La tertulia es el segundo género más empleado por Hola Chiche, seguido por el comentario y la crónica, que son casi igual de importantes si consideramos la frecuencia con la que aparecen a lo largo del programa. Todos los géneros decrecen en su uso a medida que el programa se acerca a las 13.00 horas, excepto el comentario, cuya presencia aumenta a medida que avanza el programa. Hola Chiche no suele iniciar el programa con 
comentarios, sino con participaciones más breves de los colaboradores, cuyas intervenciones son más informativas en este primer tramo y, a partir de las 11.00 horas y hasta el final del programa, son más interpretativas o de análisis.
Como se puede ver en el Gráfico 3, en El Oro y el Moro el género más empleado es la entrevista, con importante presencia en todas sus horas; seguido por el comentario en segundo lugar, que sobresale entre las 10.00 y las 12.00 horas; y por la crónica y la tertulia.

Gráfico 3. Uso de los géneros de información y opinión por hora de programa en El Oro y el Moro

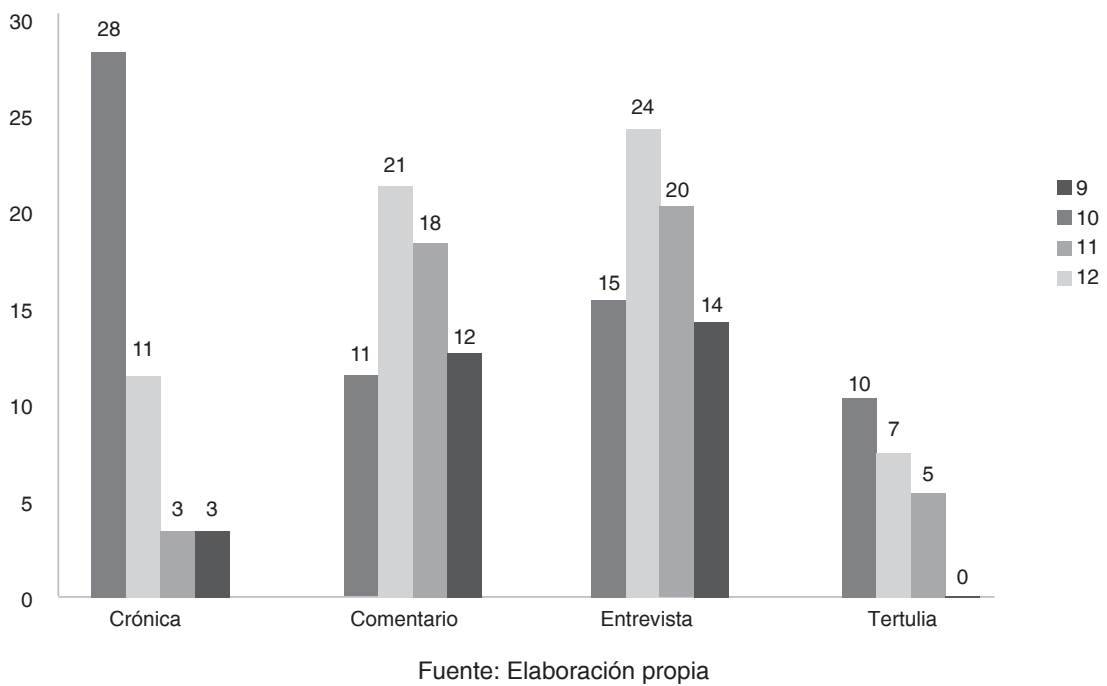

Es muy alta la presencia de la crónica en la primera hora del programa, de 9.00 a 10.00 horas, donde llega a sumar 28 en el total de la muestra. También resulta interesante observar que en $E l$ Oro y el Moro hay una mayor concentración y variedad de géneros en el tramo de las 10.00 horas.

En el caso de ¿Cuál Es? el género más utilizado es el comentario, seguido de la tertulia, como se observa en el Gráfico 4. El uso de los géneros de in- formación y opinión este programa es más irregular, incluso entre las distintas franjas horarias.

La crónica tiene muy poca presencia, igual que la entrevista. Destaca el comentario, que no se utiliza nunca en la primera hora, pero luego tiene su pico en la segunda y decrece hacia las 13.00 horas. La tertulia, en cambio, que no está muy lejos del comentario en cuanto a la cantidad, se usa especialmente en la primera y la última hora del programa. 
Gráfico 4. Uso de los géneros de información y opinión por hora de programa en ¿Cuál Es?

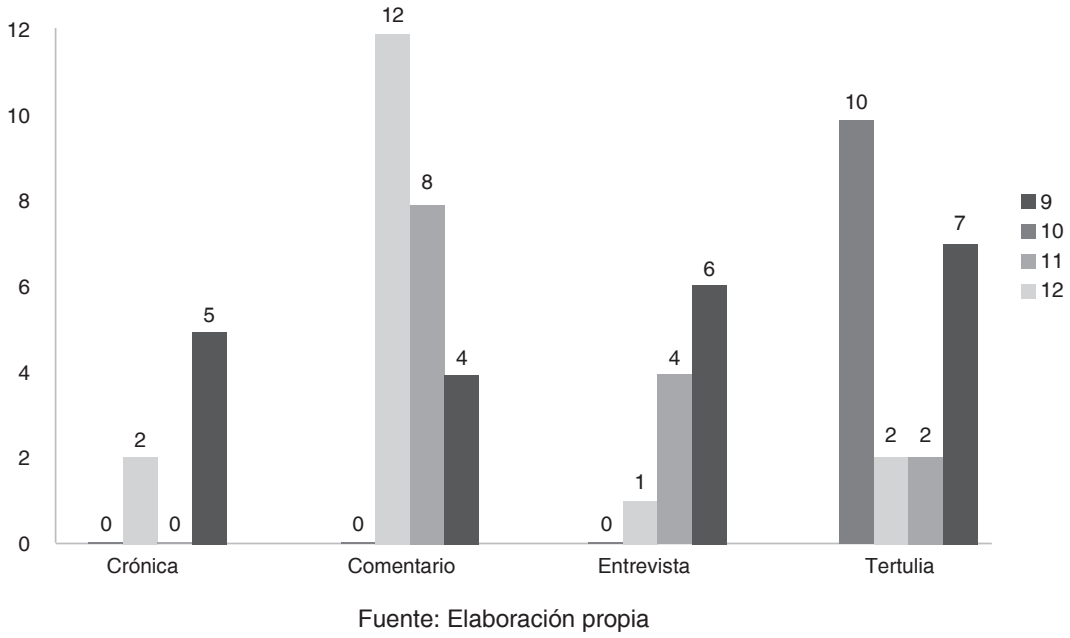

Gráfico 5. Uso de los géneros de información y opinión por hora de programa en Terapia Despareja

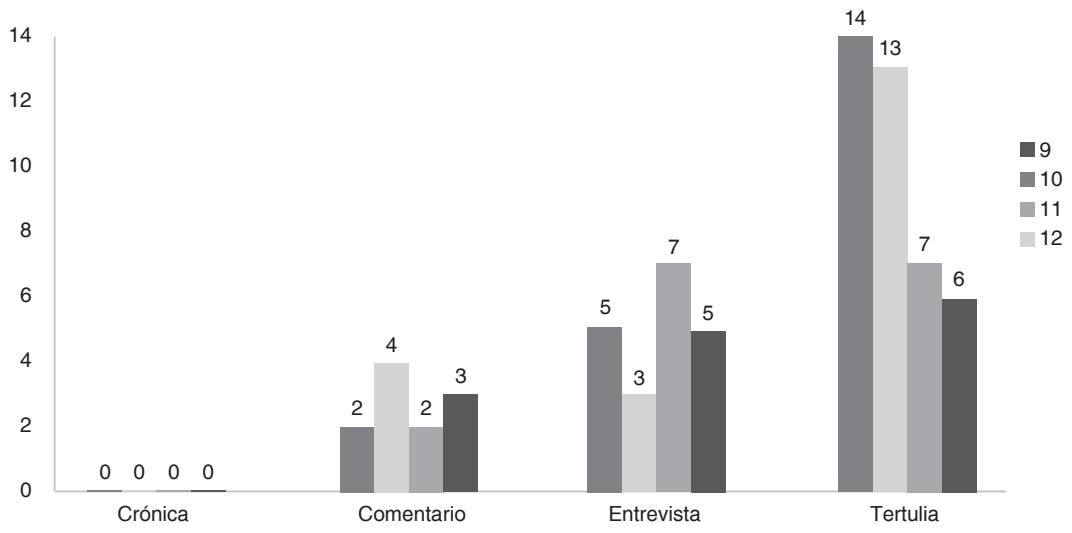

Fuente: Elaboración propia

Terapia Despareja, como muestra el gado, con dos conductores, en el que se Gráfico 5, da mayor protagonismo a la conversa y debate frecuentemente sobre tertulia en primer lugar, seguida por la distintas temáticas. Es un escenario raentrevista y el comentario. diofónico propicio para la tertulia, que

Se trata de un programa muy dialo- prevalece en las dos primeras horas del 
programa y cuyo uso desciende en las dos últimas. La entrevista no es un género muy empleado por la FM en el prime time, y Terapia Despareja presenta esporádicamente alguna entrevista en "el pase" de apertura o, sobre todo, en una sección sobre personajes que se emite los viernes. El comentario figura en tercer orden de importancia, fundamentalmente a través de un colaborador de deportes, que interviene para informar sobre la actualidad deportiva en los segmentos informativos del programa. La crónica no se utiliza en este programa.

Si analizamos el uso de los géneros de información y opinión en relación con la duración o tiempo dedicado a cada género, observamos que los resultados coinciden con los datos de frecuencia de aparición o uso: a más frecuencia de aparición, más tiempo acumulado por género, como muestra el Gráfico 6.

Gráfico 6. Tiempo acumulado de los géneros de información y opinión por programa

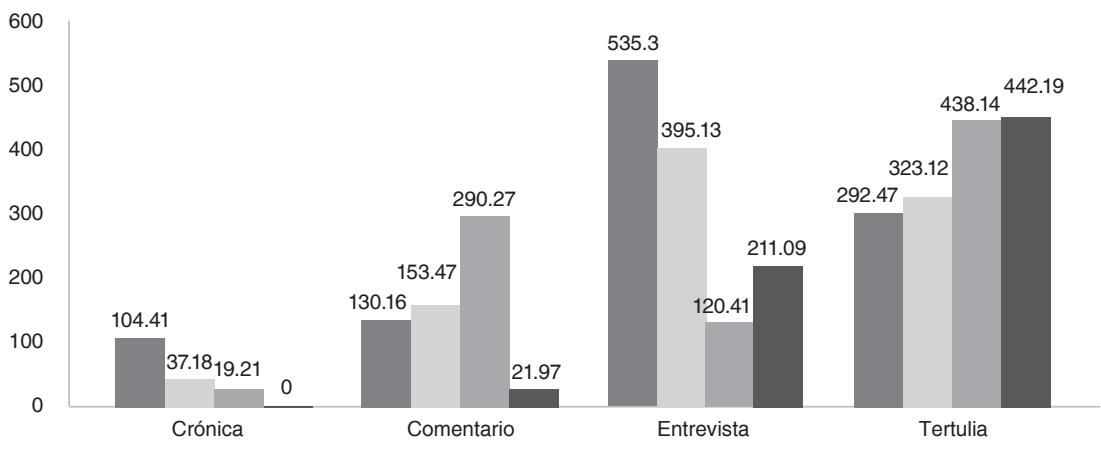

\#ola Chiche AM 790 El Oro y el Moro AM 710 Cuál es? FM $95.1 \quad$ Terapia Despareja FM 101.5

Fuente: Elaboración propia

El género en el que invierte más tiempo la OM/AM es la entrevista, mientras que la FM le dedica más minutos a la tertulia. Hola Chiche supera los 500 minutos de entrevista en el total de la muestra, y El Oro y el Moro suma casi 400. El género en el que hay más equilibrio entre los cuatro programas respecto del tiempo invertido es la tertulia. Todos los programas dedican aproxima- damente entre 300 y 450 minutos en la tertulia, lo que muestra la importancia de este género en el prime time, más allá de si se trata de OM/AM o FM. Los programas de máxima audiencia invierten menos minutos en la crónica y el comentario. La crónica es un género más propio de la OM/AM, aunque tampoco se le dedica mucho tiempo. El comentario también es más utilizado por la OM/AM 
y, como ya se señaló, El Oro y el Moro es el que ofrece mayor cantidad de comentarios. Sin embargo, ¿Cuál Es? invierte incluye comentarios de mayor duración por programa.

En resumen, los géneros de información y opinión son determinantes para el prime time de la radio argentina, si bien presentan matices en cuanto a su relevancia, frecuencia y duración. Predominan los géneros de diálogo que dan mayor ritmo a la presentación de la actualidad del día. La entrevista es el género que más diferencia a la OM/AM de la FM, en tanto que la primera lo utiliza mucho y la segunda muy poco. La tertulia, en cambio, es fundamental para todo el prime time, sin distinción entre los programas de $\mathrm{OM} /$ AM y FM. El comentario y la crónica, como la entrevista, también son más propios de la OM/AM, aunque para ¿Cuál Es?, por ejemplo, el comentario es un gé- nero relevante. En definitiva, el estudio del uso de los géneros demuestra que las propuestas programáticas entre OM/AM y FM no son tan diferentes, sino que, por el contrario, guardan muchas similitudes. Esto significa que en el prime time de la radio argentina, en lo que se refiere al uso de los géneros, la oferta de programación tiende más a homogeneizarse.

5. La utilización de los géneros de ficción y entretenimiento para el desarrollo de una radio creativa

Los géneros relacionados con la ficción y el entretenimiento que se han analizado en esta investigación son el $a c$ ting, la imitación y el cuento de humor. Se entiende por acting a todo relato de ficción corto, de no más de 5 minutos, con personajes muchas veces estereotipados, y una trama construida y mantenida casi siempre en el humor ${ }^{4}$.

Gráfico 7. Cantidad de actings por programa

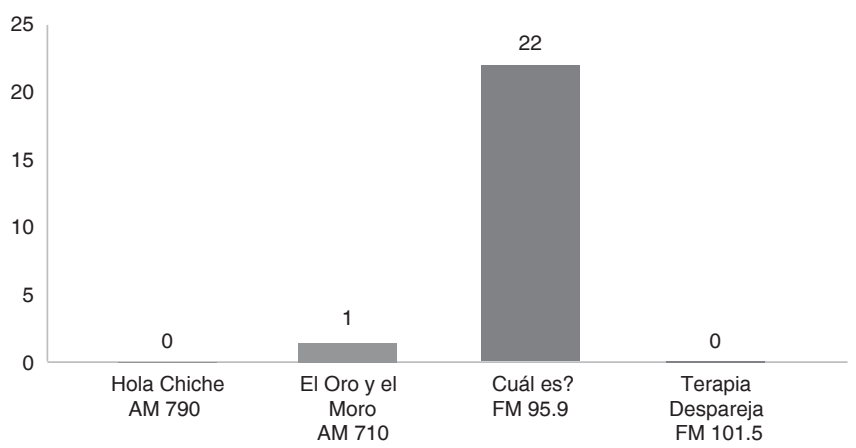

Fuente: Elaboración propia

4 Algunos autores denominan a este género sketch radiofónico, véase por ejemplo Rodero Antón (2005, 252). 
La imitación consiste en la personificación de un personaje generalmente público, a cargo de un humorista, que busca copiar y exagerar su estilo. Se denomina cuento de humor a lo que coloquialmente llamamos "chiste", un relato humorístico breve, de entre 30 y 60 segundos, con una conclusión o cierre sorpresivo y efectista.

Con respecto al uso del acting, los resultados muestran con rotundidad que se trata de un género ausente en la mayoría de los programas, con la excepción de ¿Cuál Es?

En Hola Chiche y Terapia Despareja no se utiliza el acting como género y $E l$ Oro y el Moro suma solo uno en toda la muestra. En este programa hay acting de forma esporádica, siempre durante el inicio del programa y en vivo, a cargo del humorista Rolo Villar, de González Oro o de ambos. En cambio, ¿Cuál Es? acumula 22 actings. Este re- sultado se vincula con el tipo de programa que es pero, sobre todo, con la emisora a través de la cual es emitido: Rock \& Pop. La producción artística de esta emisora y su ambiciosa preproducción dan a este género mayor prioridad para entretener a su audiencia. ¿Cuál Es? cuenta con una apertura grabada que consiste en un acting fragmentado y presentado en tres partes. Además, programa un radioteatro en vivo, con la participación de casi todos sus integrantes. Tanto la apertura como el radioteatro son dos secciones emblemáticas del programa, que sus oyentes identifican claramente.

El segundo género de ficción y entretenimiento analizado es la imitación. El Gráfico 8 demuestra que su importancia presenta grandes variaciones según cada programa, aunque está presente en todos.

Gráfico 8. Cantidad de imitaciones por programa

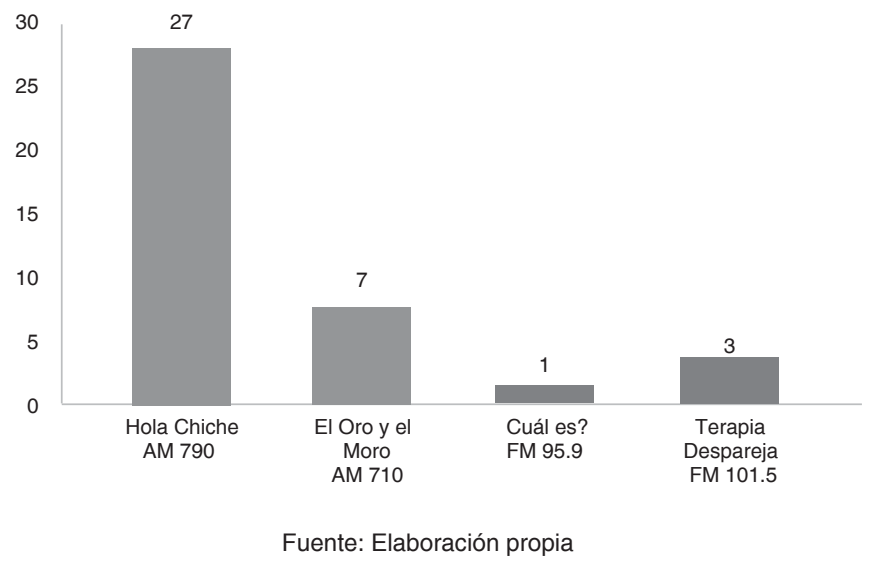


Gráfico 9. Cantidad de cuentos de humor por programa

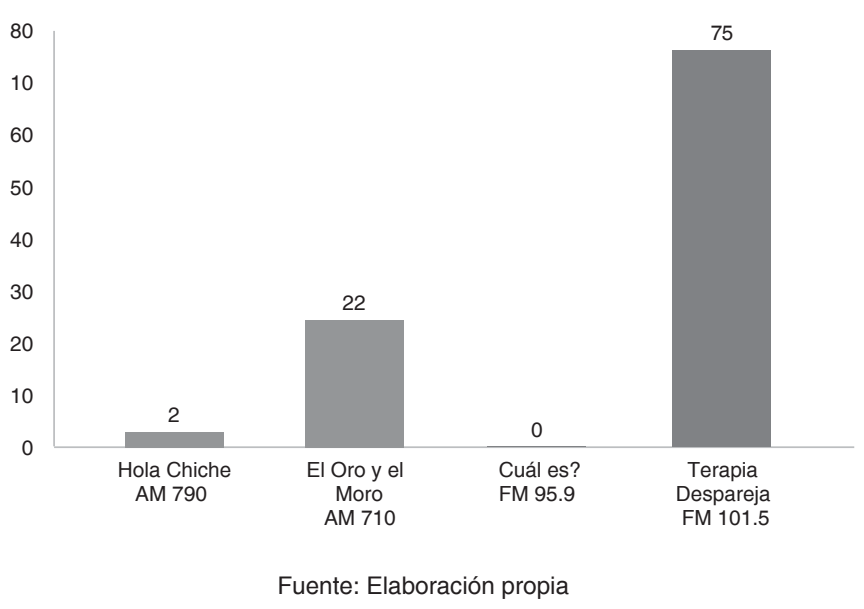

Está claro que la imitación ocupa un lugar en Hola Chiche muy diferente al resto de los programas. Cuenta con un humorista que es, sobre todo, imitador y por ello la mayoría de sus intervenciones cómicas son imitaciones. Desarrolla distintos personajes según los temas de actualidad que se aborden. Por ejemplo, en los días que forman parte de la muestra de esta investigación, destaca la imitación del entonces presidente chileno Sebastián Piñera, que tiene como contexto el relato del rescate de los 33 mineros atrapados en la mina San José. El Oro y el Moro, aunque con mucha menor frecuencia, es el programa que ocupa el segundo lugar en el uso de este género de entretenimiento. Las esporádicas imitaciones de este programa están a cargo de Rolo Villar, humorista que interviene el arranque del programa o el "pase" entre El Oro y el Moro y el pro- grama que lo precede: Cada Mañana. En tercer y cuarto lugar figuran Terapia Despareja, con 3 imitaciones, y ¿Cuál Es?, con una.

El cuento de humor, como se refleja en el Gráfico 9, es un género que algunos programas utilizan con mucha frecuencia mientras que otros prescinden de él o lo usan de modo esporádico.

El cuento de humor -o también llamado "chiste"- resulta estratégico en el modo de entretener de Terapia Despareja. Rolo Villar, humorista del programa, remata muchos de los bloques con un cuento. Son muy breves y casi siempre relativos al tema del que se está hablando. Por esto, Terapia Despareja saca ventaja sobre el resto con 75 cuentos de humor. Detrás aparece El Oro y el Moro, que suele dar espacio a los "chistes" solo en el "pase" o transición con el programa anterior, Cada Mañana. Hola Chiche se ubica 
tercero, con solo dos cuentos, porque, como se anticipó, su humorista entretiene fundamentalmente a través de imitaciones.

Se puede concluir que los programas establecen un criterio propio a la hora de utilizar los géneros de ficción y entretenimiento en el prime time. Así, un género como el acting, que supone más producción, prevalece en la FM y en un programa con más tratamiento artístico del estilo de ¿Cuál Es?. Las imitaciones y los cuentos de humor son tan propios de la OM/AM como de la FM en la segunda mañana. Terapia Despareja se vale de los cuentos para cerrar los bloques y Hola Chiche entretiene, sobre todo, a través de las imitaciones de Ariel Tarico. El único programa que emplea más esporádicamente estos géneros es $\mathrm{El}$ Oro y el Moro. En todos los programas, se ve claramente que el uso de géneros de ficción y entretenimiento, tanto de forma planificada como espon- tánea, ayuda a marcar los cambios de ritmo de las diferentes horas del programa.

\section{Los géneros de participación dina- mizan la puesta en antena}

Los géneros de participación de la audiencia analizados fueron los concursos y los mensajes, ya que aparecen con mayor frecuencia. Los concursos son espacios de interacción con el oyente, quien es interpelado a participar para obtener un premio mediante la respuesta a una consigna. Los mensajes son las comunicaciones que los oyentes hacen llegar al programa a través de correos electrónicos, redes sociales o mediante la grabación su voz.

En cuanto a la cantidad de concursos que hay en cada programa, se observa que la FM propone más concursos que la OM/AM. El programa que lidera la cantidad de concursos es Terapia Despareja, con 20, seguida por ¿Cuál Es?, con 11, y por El Oro y el Moro, con 6.

Gráfico 10. Cantidad de concursos por programa

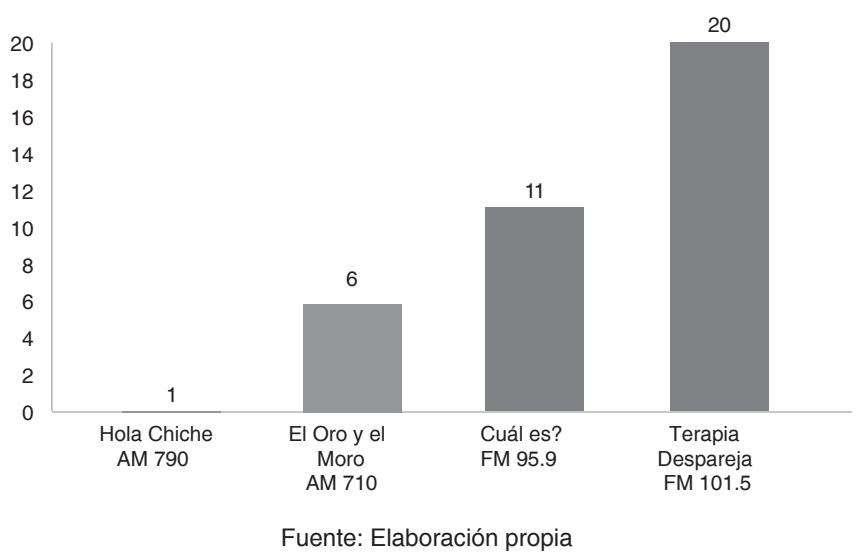


Hola Chiche, al menos en el período estudiado, no utiliza el concurso. Es interesante destacar además que el concurso se concentra, sobre todo, en la última franja horaria, de 12.00 a 13.00 horas.

Queda en evidencia que casi no se programan concursos en la primera hora. Recién a partir de la 10.00 horas los programas empiezan a incentivar la participación de su audiencia a través de concursos. En el horario de 10.00 a
11.00 horas y de 11.00 a 12.00 horas se proponen el mismo número de concursos (8), y esa cifra prácticamente se triplica en la última franja horaria. Estos resultados revelan que el entretenimiento a través de concursos se planifica para el último tramo de los programas.

Los mensajes son el otro modo de interacción con la audiencia analizado en este trabajo. En el Gráfico 12 se distingue la cantidad de mensajes por programa.

Gráfico 11. Cantidad de concursos por hora de programa

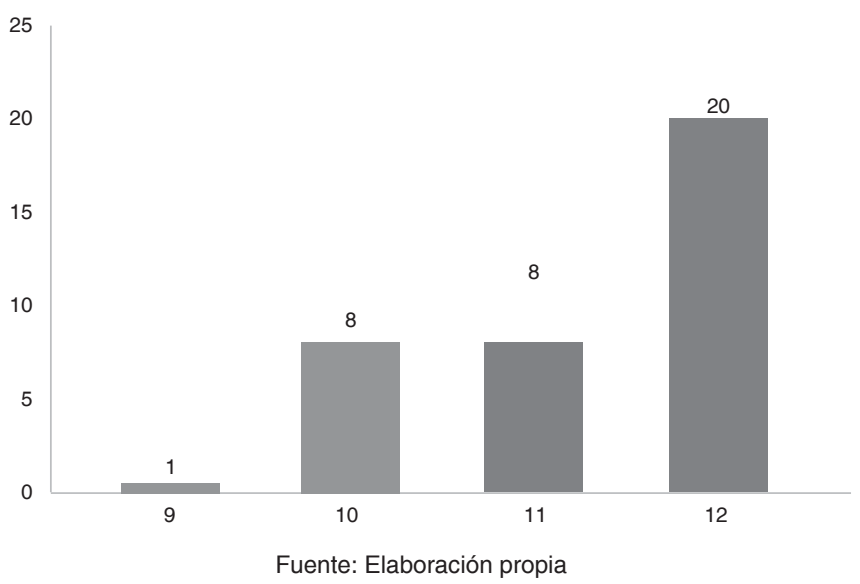

Terapia Despareja destaca en la interacción con su audiencia a través de mensajes. Suma 78, seguido por El Oro y el Moro, con 48. Luego aparecen Hola Chiche y ¿Cuál Es? con 29 y 21 mensajes, respectivamente. Esto muestra que no hay similitudes entre las emisoras del mismo soporte, de modo de que cada programa tiene su propio estilo en el uso de los mensajes. Para Terapia Despareja resulta un contenido prioritario, sobre los que construye el discurso.
Los mensajes impulsan los temas que se abordan, aportan nuevos, instalan oyentes que se convierten en personajes a fuerza de su originalidad y humor. En definitiva, producen contenido y dinamizan el directo. En el caso de $E l$ Oro y el Moro, la mayoría de los mensajes se emiten grabados, siempre tras la tanda y sobre un tema musical que utilizan como sintonía de fondo. También el conductor y la locutora leen mensajes que llegan a través de correos 
electrónicos o de las redes sociales. En der la mayoría de los mensajes, casi Hola Chiche y en ¿Cuál Es? los conducto- siempre con la intención de generar pores son los encargados de leer y respon- lémica y evitar adulaciones o saludos.

Gráfico 12. Cantidad de mensajes por programa

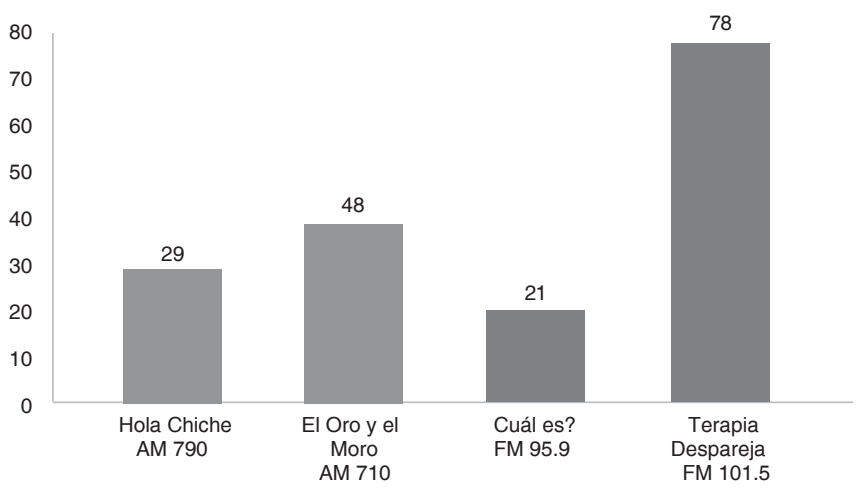

Fuente: Elaboración propia

Al cruzar los datos según las franjas mensajes en las horas intermedias, y horarias, podemos ver en el Gráfico 13 menos en las franjas de 9.00 a 10.00 hoque todos los programas tienen más ras y de 12.00 a 13.00 horas.

Gráfico 13. Cantidad de mensajes por programa y franja horaria

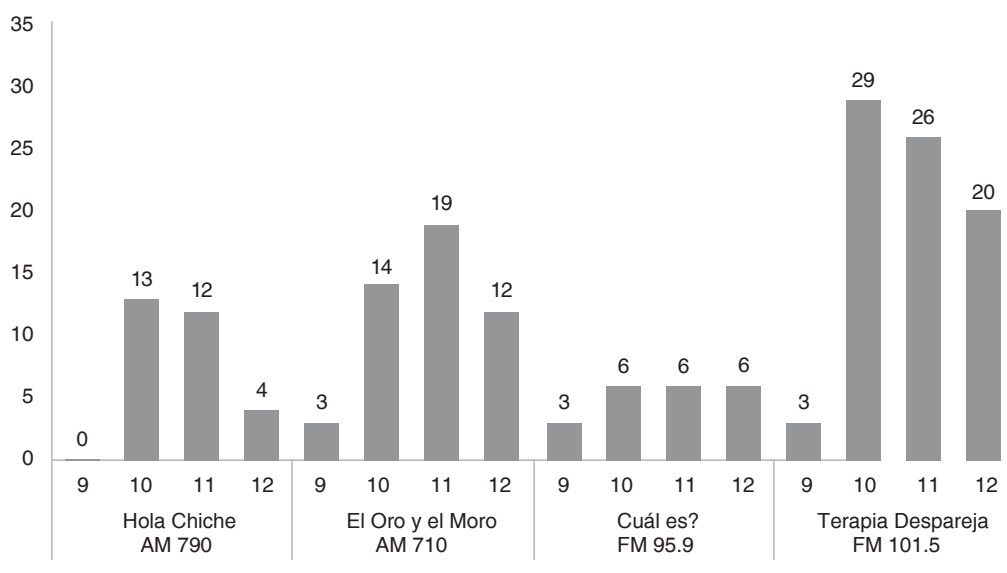

Fuente: Elaboración propia 
Es razonable que la primera hora acumule menos mensajes de oyentes. Éstos surgen generalmente a partir de lo que está pasando en el programa y lo retroalimentan. Los programas también coinciden en un decrecimiento en la última hora, excepto ¿Cuál Es?, que mantiene la misma cantidad que en las franjas que van de 10.00 a 11.00 horas y de 11.00 a 12.00 horas.

En resumen, cada programa encuentra su propia forma de interactuar con el oyente. Todos utilizan los mensajes como modo de participación, no así los concursos, que son un recurso de acercamiento al oyente que emplean con más diferencias entre sí. Terapia Despareja es el que acumula más concursos y mensajes, lo que lo define como el programa con mayor interacción con el oyente. Todos coinciden en programar los concursos más próximos al cierre del programa y en dar más espacio a los mensajes en las horas intermedias: de 10.00 a 11.00 horas y de 11.00 a 12.00 horas.

\section{Conclusiones}

El concepto de programa magacín como un programa contenedor de larga duración, con voces y temáticas variadas que fluyen en un modo de contar sin aparente orden y con la espontaneidad del diálogo, no es incompatible con la identificación y delimitación de los géneros radiofónicos utilizados.

Tras realizar el estudio, se comprueba que, aunque predomina la conversación aparentemente no planificada -dirigida por un presentador estrella sobre el que pivota todo el discurso-, el uso de los géneros radiofónicos en los programas de máxima audiencia es frecuente, se puede delimitar y se pueden identificar algunos como predominantes.

Con respecto al uso de los géneros de información y opinión, el estudio revela que las propuestas programáticas entre $\mathrm{OM} / \mathrm{AM}$ y $\mathrm{FM}$ no son tan diferentes, sino que, por el contrario, responden a estrategias similares para ganar la confianza y la fidelidad de la audiencia. Hay mayor diferencia en la presencia de la entrevista y la crónica -que son géneros informativos-, pero menos en la tertulia y el comentario -que son géneros de opinión- tanto en la frecuencia como en la duración. Por este motivo, en la utilización de este tipo de géneros, la oferta de programación del prime time de la radio argentina tiende más a homogeneizarse que a diferenciarse entre la OM/AM y la FM.

En la producción de ficción y entretenimiento, destacan inicialmente los programas de la FM que dedican desde siempre más recursos a la producción artística. Esto es muy claro en la utilización del acting, pero se diluye en el caso de las imitaciones y los cuentos de humor: el análisis muestra que son tan propios de la OM/AM como de la FM en la segunda mañana de la radio argentina, cuando los contenidos van abandonando progresivamente la actualidad de la hora como eje principal de la conversación para abrirse a otros temas.

Además, cada programa diseña su propia forma de interactuar con el oyente. Sigue siendo muy habitual la lectura o 
escucha de mensajes directos de la audiencia, como modo de participación, sobre todo en las horas intermedias de cada programa. Menos utilizados son los concursos, que son un recurso de acercamiento al oyente que cada programa emplea con una frecuencia muy diferente, aunque todos coinciden en programarlos más próximos al cierre de cada edición del programa.

Por tanto, se puede afirmar como conclusión general que la oferta de tratamientos narrativos de la OM/AM y de la FM en las horas de máxima audiencia de la radio argentina es bastante homogénea. Así, la producción y tratamiento periodístico de la actualidad que han sido el contenido predominante de OM/AM, forma parte también de la apuesta de FM; mientras que el cuidado artístico y creativo de las formas de contar propiciado por la calidad sonora de la FM, ahora también se hace presente en la OM/AM. Como se ha visto, esta tendencia es más marcada en el tramo de la segunda mañana de la radio, donde se concentra la mayor audiencia. Todos los programas analizados asumen las peculiaridades de los magacines tradicionales de la radio generalista de OM/AM, al tiempo que cuidan la producción artística y los ritmos narrativos propios de la FM. De esta forma facilitan la variedad de los modos de contar, haciendo uso de una amplia gama de géneros de información, opinión, ficción, entretenimiento y participación, con el fin estratégico de ganar y consolidar la audiencia.

Aunque la denominación de los programas y los conductores cambian, esta estructura y combinación de los modos de contar se mantiene actualmente en los programas más escuchados de la radio en Argentina. El estudio realizado, por tanto, se constituye en un punto de partida para comparar con la realidad de otros mercados de la radio, así como para analizar su evolución en futuras investigaciones.

\section{Bibliografía}

Cuní, J. (1999). La ràdio que triomfa. Barcelona: Portic.

Fleming, C. (2010). The Radio Handbook (Third edition). Londres: Routledge.

Haye, R. (1995). Hacia una nueva radio. Buenos Aires: Paidós.

Haye, R. (2003). Otro siglo de radio. Noticias de un medio cautivante. Buenos Aires: La Crujía.

López Vigil, J.I (1997). Manual urgente para radialistas apasionados. Quito: Silva.

Martí, J. (2000). De la idea a l antena: tecniques de programació radiofonica. Barcelona: Portic. Martínez-Costa Pérez, M. P.; Díez Unzueta, J. R. (2005). Lenguaje, Géneros y programas de radio, Introducción a la Narrativa Radiofónica. Pamplona: Eunsa.

Martínez-Costa Pérez, M. P.; Herrera Damas, S. (2004). Los géneros radiofónicos en la teoría de la redacción periodística en España. Luces y sombras de los estudios realizados hasta la actualidad. Comunicación y Sociedad 12 (1), pp. 115-143.

Martínez-Costa Pérez, M. P.; Herrera Damas, S. (2005). Qué son los géneros radiofónicos y por qué deberían importarnos. Global Media Journal 2(3). Disponible en:

http://gmje.mty.itesm.mx/articulos3/articulo_7.html. Consultado el 6 de mayo de 2013. 
Martínez-Costa, M. P.; Moreno, E.; Amoedo, A. (2018). El mapa de la radio online en España: tipología y caracterización en el contexto de los cibermedios. El profesional de la información, 27 (4), en prensa.

Martínez-Costa, M. P.; Müller, M.J. (2015). Content and Audience of Morning 'Magazine' Radio Programs in Argentina and Spain. The International Journal of Hispanic Media, 8, pp. 24-37.

Moreno, E.; Amoedo, A.; Martínez-Costa, M.P. (2017). Usos y preferencias del consumo de radio y audio online en España: tendencias y desafíos para atender a los públicos de internet. Estudios sobre el mensaje periodístico 23 (2), 1319-1336. Disponible en: http://revistas. ucm.es/index.php/ESMP/article/view/58047/52232.

Noguer, J. (1985). Radiodifusión en la Argentina. Buenos Aires: Editorial Bien Común.

Portugal, M.; Yudchak, H. (2008). Hacer radio. Guía integral: cómo se hace un programa de radio, paso a paso. Buenos Aires: Galerna.

Rodero Antón, E. (2005). Producción radiofónica. Barcelona: Cátedra.

Rodero Antón, E. (2011). Creación de programas de radio. Madrid: Síntesis.

Ulanovsky, C. (1995). Días de radio. Buenos Aires: Espasa Calpe. 